Portland State University

PDXScholar

$5-1-1992$

\title{
Transmission studies of explosive vaporization of a transparent liquid film on an opaque solid surface induced by excimer-laser-pulsed irradiation
}

\author{
P.T. Leung \\ Portland State University \\ Nhan Do \\ Leander Klees \\ Wing P. Leung \\ Frank Tong
}

Follow this and additional works at: https://pdxscholar.library.pdx.edu/phy_fac

Part of the Physics Commons

Let us know how access to this document benefits you.

\begin{abstract}
Citation Details
Leung, P. T., Do, N., Klees, L., Leung, W. P., Tong, F., Lam, L., \& ... Tam, A. C. (1992). Transmission studies of explosive vaporization of a transparent liquid film on an opaque solid surface induced by excimer-laserpulsed irradiation. Journal Of Applied Physics, 72(6), 2256.
\end{abstract}

This Article is brought to you for free and open access. It has been accepted for inclusion in Physics Faculty Publications and Presentations by an authorized administrator of PDXScholar. Please contact us if we can make this document more accessible: pdxscholar@pdx.edu. 


\title{
Transmission studies of explosive vaporization of a transparent liquid film on an opaque solid surface induced by excimer-laser-pulsed irradiation
}

\author{
P. T. Leung, ${ }^{\text {a) }}$ Nhan Do, ${ }^{\text {b) }}$ Leander Klees, ${ }^{\text {c) }}$ W. P. Leung, ${ }^{d)}$ Frank Tong, ${ }^{e)}$

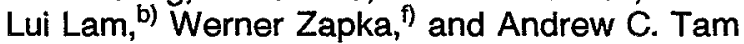 \\ IBM Research Division, Almaden Research Center, 650 Harry Road, San Jose, California 95120-6099
}

(Received 6 February 1992; accepted for publication 26 May 1992)

\begin{abstract}
The dynamics of the explosion of a liquid film by an ultraviolet excimer pulsed laser is studied experimentally on top of an amorphous silicon ( $a$-Si) film deposited on fused quartz. In particular, the transient temperature as well as the nucleation of the superheated liquid at the interface for laser fluences below and above the spontaneous nucleation threshold are monitored using an optical transmission technique. Results for different pure and mixed liquids are presented for cases of different thicknesses of the $a$-Si film. Details for the calibration of the transmission signal to obtain the interface temperature are provided through the application of the one-dimensional diffusion model and the characteristic matrix theory using data obtained from previous steady-state experiments. The calibrated results are in reasonable agreement with the known data for superheated liquids in the literature as well as theoretical calculations. In addition, the delay times for spontaneous nucleation of the liquids are estimated to be $\lesssim 1 \mu \mathrm{s}$, generally comparable with those observed in droplet explosion experiments.
\end{abstract}

\section{INTRODUCTION}

The thermal physics of superhcated pure or mixed liquids has been an area of interest for many years. Questions such as the universal behavior, kinetic or thermodynamic superheat limits, and possible supercritical phenomena concerning those metastable systems have been studied extensively in the literature. ${ }^{1}$ It is well known that one of the efficient ways to create such a metastable system is through pulsed heating of the liquid. While pulsed electric current heating can usually be achieved in the microsecond time scale, short pulsed-laser heating can provide a much wider range of time scale for the study of the superheating of the liquids. In particular, submicrosecond heating of the free surface of a liquid in a container ${ }^{2}$ as well as free liquid droplet $^{3}$ has been studied in the literature. In all these cases, the laser wavelength is in the absorptive region of the liquid and the heating is direct. In this work, we present a study on the indirect superheating of a transparent liquid at the interface of an absorbing substrate surface in the nanosecond time scale. The motivation of the present work also comes from recent application of the huge pressure that can be generated by these superheated liquids for cleaning as discussed below.

With the increasing importance of removing submicrometer particles (contaminants) from substrate surfaces

\footnotetext{
${ }^{a)}$ Present address: Department of Physics, Portland State University, Portland, OR 97207-0751.

b) Present address: Department of Physics, San Jose State University, San Jose, CA 95192-0106.

${ }^{c}$ Present address: Institut fur Angewandte Physik, Technische Hochschule Darmstadt, Hochschulstr. 6, D-6100 Darmstadt, Germany.

d) Present address: IBM, F12A/013, 5600 Cottle Road, San Jose, CA 95193.

e)Permanent address: IBM Research Division, T. J. Watson Research Center, Yorktown Heights, NY 10598.

f) Permanent address: IBM Deutschland, GMTC, 7031 Sindelfingen, Germany.
}

in the electronics industry, the technique of laser cleaning has been actively developed by various groups in the last few years. ${ }^{4-10}$ This approach includes method to heat up the particle, ${ }^{4}$ the substrate surface, ${ }^{5,6}$ as well as the liquid film $\mathrm{m}^{5,7-10}$ in the case when such a film is deposited on the surface to facilitate the cleaning process. Recent studies by us have shown that one of the most efficient ways to achieve laser cleaning of surfaces is to deposit a transparent liquid film (e.g., alcohol-water mixture) and to heat up the liquid-substrate interface with a UV excimer pulsed laser. ${ }^{7}$ This technique can allow one to remove particles down to $0.1 \mu \mathrm{m}$ in size from the substrate surface using a modest fluence of about $0.1 \mathrm{~J} / \mathrm{cm} .{ }^{8}$ However, in spite of the success of the method, the detail of the mechanism of this approach has yet to be understood. It is expected that a thin layer of the liquid $(\sim 0.1 \mu \mathrm{m})$ at the interface must have been strongly superheated during the short duration $(\sim 10$ ns) of the laser pulse; explosion then takes place and particles are ejected from the surface by the huge liquid pressure due to explosion. Since the damage of the substrate surface is very sensitive to the temperature and pressure of the superheated liquid, the monitoring and control of these parameters become very important for both the understanding of the process and the protection of the substrate surface from damage.

In this paper, we study the nucleation dynamics of the exploding liquid film mentioned in the above process. In particular, data from the measurement of the liquid-solid interface temperature will be presented and compared with those for superheated liquids in the literature as well as those from theoretical calculations. We have developed an optical transmission technique for such temperature measurements and have chosen amorphous silicon $(a$-Si) as our substrate surface. The organization of the paper is as follows. We shall start first to elaborate the rationale of our approach to this problem in Sec. II, then the experimental details will be presented in Sec. III, with results and anal- 
ysis presented in Secs. IV and V, and conclusions in Sec. VI. We shall see that in addition to the interface temperature, one can also give an estimate for the explosion time of the superheated liquid layer to be $\$ 1 \mu \mathrm{s}$, in general agreement with the values reported from experiments on explosion of liquid droplets. ${ }^{11}$

\section{APPROACH}

While there are many existing techniques for surface temperature measurement, optical reflection or transmission technique remains one of the most attractive options for its noncontact nature and fast time response capability. This technique is based on the variation of the optical properties of the substrate surface at elevated temperatures. Depending on the probe frequency and the substrate, this effect has been well studied in the literature for various metallic, ${ }^{12}$ semiconducting, ${ }^{13}$ and insulating ${ }^{14}$ substrate materials. Although the effect for the reflection from the single air-substrate interface is generally very small [e.g., $d \mathscr{R} / d T \sim 10^{-5} \mathrm{~K}^{-1}$ for crystalline silicon (c-Si) (Ref. 15)], it can be much larger if the sample is deposited in the form of a thin film on an insulator substrate due to interference effects. One of the most well-known examples for the later case is SOS ( $c$-Si on sapphire) which has been studied extensively in the literature. ${ }^{16}$ Also in this thin-film case, both reflection and transmission can be monitored for a large range of probe wavelength. As for the single interface reflectance approach, most previous studies were limited to situations where there is phase transition (such as when the substrate surface is molten) in which a large change of reflectance (or transmittance) occurs. Since our expected range of temperature to be measured is somewhere from room temperature to about $400^{\circ} \mathrm{C}$ for the liquid (recall that the critical temperature of water is $374^{\circ} \mathrm{C}$ ), which is far below the melting point of substrates like $\mathrm{Si}^{15}$ we have decided to adopt the thin-film interference approach. Previous investigations using this approach have achieved steady-state ${ }^{17}$ and millisecond ${ }^{18}$ time-resolution temperature measurements, in which uniform heating of the sample can be assumed. Bloembergen and co-workers ${ }^{19}$ have studied picosecond pulse heating of SOS and the average temperature of the very thin $(0.1 \mu \mathrm{m}) c$-Si film was obtained by considering nonuniform temperature distribution inside the Si film. In the following, we will consider a similar temperature profile effect for our nanosecond pulse heating, and our main interest is to obtain the surface temperature of the substrate. More importantly, we believe that this present work is the first time that the dynamics of a heated liquid film is studied on such thin-film structures using optical probing techniques. ${ }^{20}$ Furthermore, we have chosen amorphous silicon as our substrate surface to study for the following reasons. As noted above, while structures like $c$-Si on insulator have been studied extensively for surface heating phenomenon, it seems that, to our knowledge, $a$-Si on insulator has not been studied for transient surface heating phenomenon, except when there is surface melting that may lead to recrystallization and that occurs at a much higher-temperature regime. ${ }^{21}$ So it would be of interest to study transient heating of $a-\mathrm{Si}$ at a much lower temperature range and to compare it with the case for $c-\mathrm{Si}$. We shall see that to achieve the same elevated surface temperature, the UV laser fluence required is much lower in the case of $a$-Si compared to that of $c$-Si. In addition, the preparation of the $a$-Si film on insulating substrate is relatively easier, and we expect that while the heating details (e.g., the interface temperature for a given laser fluence) will depend on the properties of the substrate surface, the nucleation dynamics of the liquid will be quite general on any opaque surface of comparable surface finish. Hence the results we obtain in this later aspect should have some validity if other kinds of substrate surfaces such as $c$-Si are used in the experiment.

There have been some indications in the literature ${ }^{22}$ that the optical constants of $a$-Si are very insensitive to temperature change. This will render a transmission monitoring approach impossible since we look for the change of optical properties when the $a-\mathrm{Si}$ surface is heated by the excimer pulse. However, in a more thorough study, ${ }^{23}$ we have concluded that the previous results ${ }^{22}$ are incomplete and are true only for an $a$-Si sample film probed at a highly transparent wavelength. With an optimization of the two parameters, sample thickness and probe wavelength, one can indeed see large temperature effects in the optical properties of $a-\mathrm{Si}$. We are thus confirmed that our approach to the problem of transient surface temperature measurement for $a$-Si is indeed possible and, in fact, we shall use our previous steady-heating experimental data ${ }^{22}$ for calibration of the present results in the following.

\section{EXPERIMENT}

The $a$-Si samples for our experiment are prepared by $e$-beam evaporation of $c$-Si in vacuum ( $\left.10^{-7} \mathrm{Torr}\right)$ onto fused quartz substrates ( $250 \mu \mathrm{m}$ thick $)$. The substrate temperature is kept at $140^{\circ} \mathrm{C}$ and the deposition rate at 10 $\AA / s$. Different thicknesses of $a$-Si are prepared by controlling the deposition time, the uniformity, and accuracy of these thicknesses are confirmed using the Tencor AlphaStep 200 surface profilometer. The samples are then annealed at a temperature of $380^{\circ} \mathrm{C}$ in a nitrogen chamber for about $5 \mathrm{~h}$ until optical measurements (reflectance and transmittance) versus change of temperature are reproducible. Under these conditions, the formation of oxide or nitride is kept at a minimum. ${ }^{24}$ Stress relaxation of the sample is observed due to the above heat treatment.

Our experimental setup is shown in Fig. 1. The sample is mounted with the $\mathrm{KrF}$ excimer laser ( $248 \mathrm{~nm}, \sim 16 \mathrm{~ns}$ ) pulse incident at a small angle $\left(\sim 5^{\circ}\right)$ and the cw probe diode laser $(752 \mathrm{~nm}$ ) going through it at normal incidence. In case when reflectance is also monitored, the probe laser is incident at a small angle $\left(\sim 10^{\circ}\right)$. The temporal evolution of the transmission signal is obtained by a fast photodiode and a digitalizing oscilloscope (HP 54111D). The energy fluence is determined by measuring the pulse energy using an energy meter (Molectron JD 1000) with the area of the UV beam measured to be $\sim 0.5 \mathrm{~cm}^{2}$. The liquid layer is deposited onto the sample using a homemade puffer which is described elsewhere. ${ }^{7}$ The time delay (typically $0.1 \mathrm{~s}$ ) between the puffer and the firing of the UV pulse is 


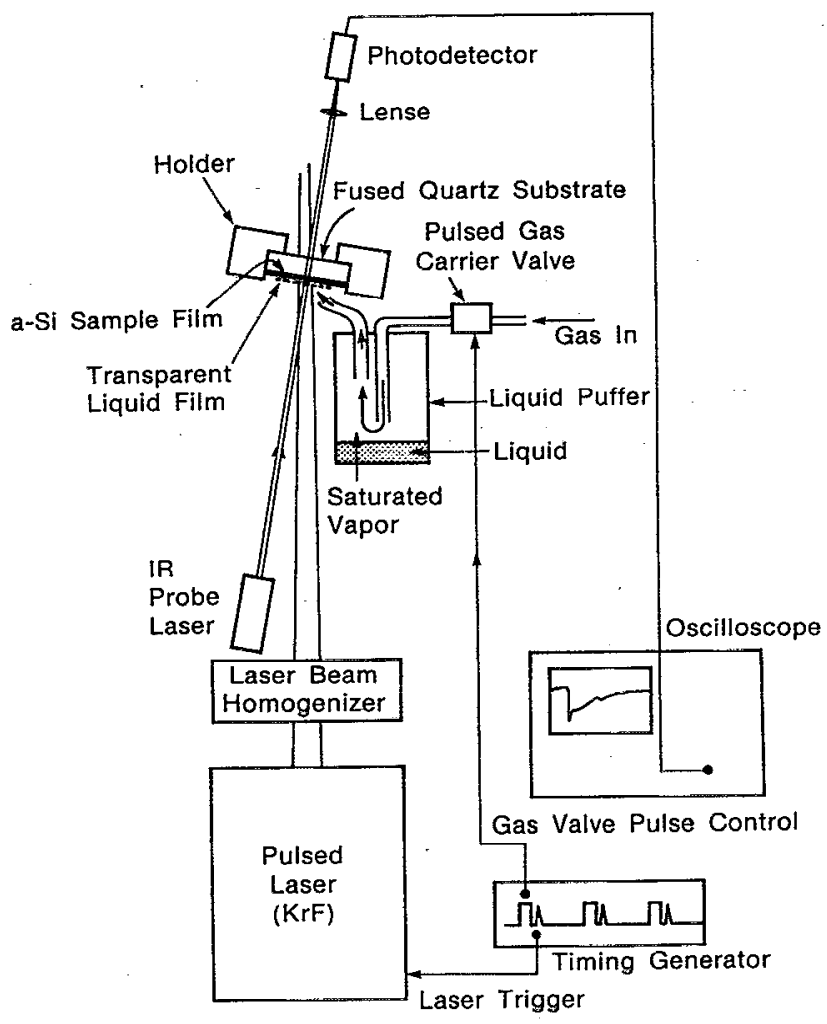

FIG. 1. Experimental setup shown with the 752-nm IR probe laser and the 248-nm pulsed excimer laser.

controlled by the timing generator which first turns on the gas flow used in the puffer and then triggers the firing of the $\mathrm{KrF}$ laser.

\section{RESULTS AND DISCUSSIONS}

Some typical signals are shown in Figs. 2 and 3 for two $a$-Si films of different thicknesses $(0.2$ and $1.0 \mu \mathrm{m})$ used in our experiment. Figure 2(a) shows that the transmittance $(\mathscr{T})$ and reflectance $(\mathscr{R})$ of the probe diode laser are complementary to each other, in response to the UV pulse irradiating at the bare (dry) surface of the $0.2-\mu \mathrm{m} a-\mathrm{Si}$ film. Figure 3(a) shows a similar behavior in $\mathscr{T}$ for the $1.0-\mu \mathrm{m}$ film. Unlike the large transmission drop observed in previous experiments ${ }^{16}$ which was due to the phase transition of the Si sample, these drops in $\mathscr{T}$ are due exclusively to the increase in absorption of the $a$-Si films caused by the rise in temperature. Figures $2(\mathrm{~b})-2$ (f) show the signals for a wetted surface with different liquids used. For simplicity, the $\mathscr{R}$ signal is only shown for the isopropanol case. In Fig. 2(b), the inset shows a long-time evolution of $\mathscr{T}$ for the probe laser going through an isopropanol film (of micrometers thickness) deposited on the $0.2-\mu \mathrm{m} a-\mathrm{Si}$ film. Both the deposition and the evaporation phases for the liquid can be easily identified from the different periods in the interference fringes. The arrow in the inset indicates the drop in $\mathscr{T}$ in response to the UV pulse; this is magnified in the same figure in the nanosecond time scale together with the corresponding signal for $\mathscr{R}$. From these
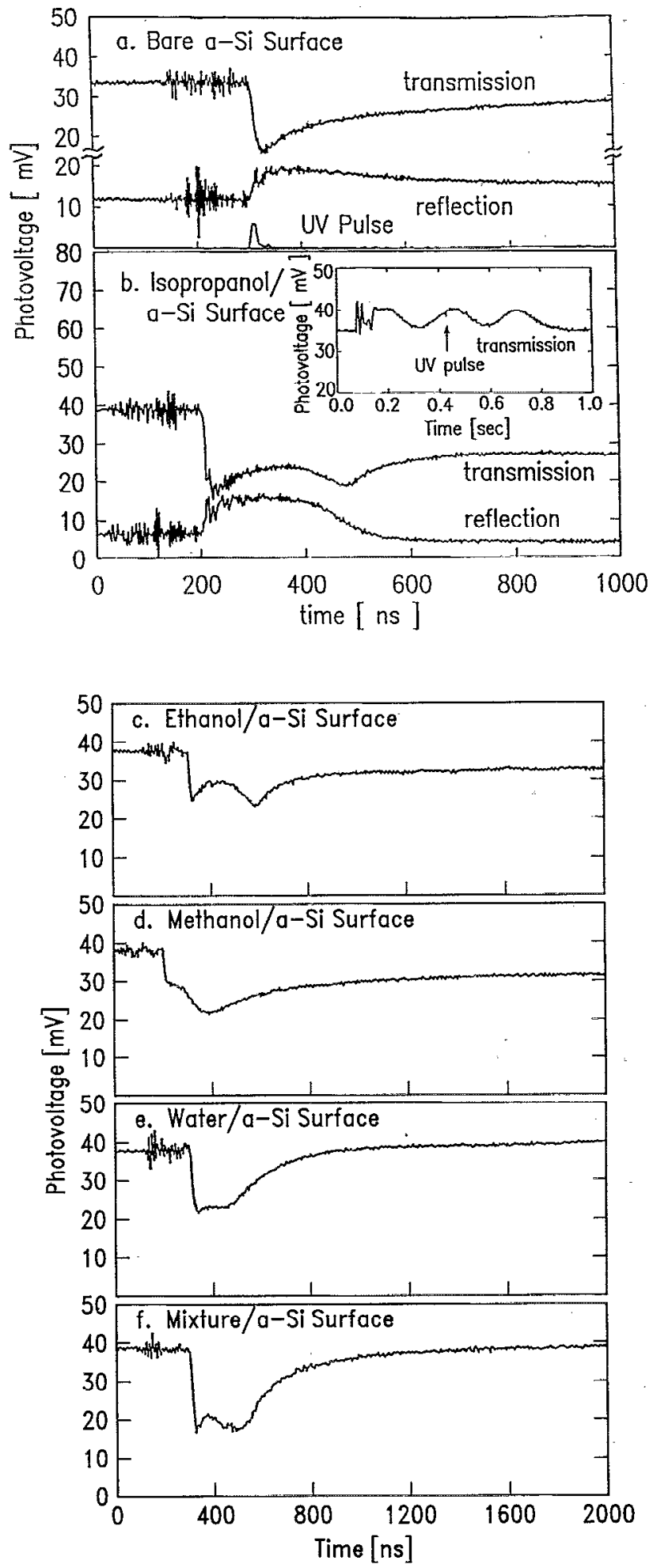

FIG. 2. Typical data for the probe transmission and refiection for the $0.2-\mu \mathrm{m} a-\mathrm{Si}$ sample. Note the different time scales for (a) and (b) as compared to that of (c)-(f). The "mixture" for (f) refers to $70 \%$ water and $30 \%$ isopropanol.

signals, we can make the following observations: (1) The drop in $\mathscr{T}$ close to the end of the UV pulse indicates the temperature rise at the liquid-solid interface as in the case of bare surface; (2) an additional second dip in $\mathscr{T}$ appears 

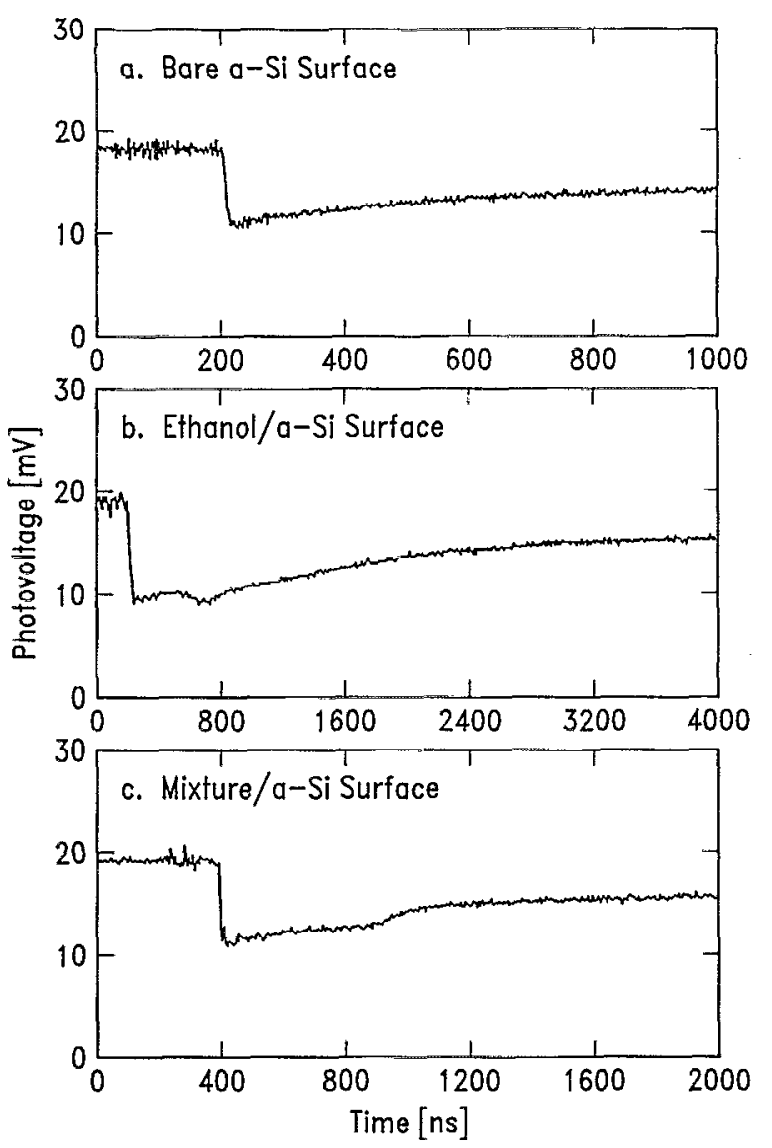

FIG. 3. Typical data for the probe transmission for the $1.0-\mu \mathrm{m} a-\mathrm{Si}$ sample. Note the different time scales for (a), (b), and (c). The "mixture" in (c) refers to $70 \%$ water and $30 \%$ isopropanol.

for laser fluence above $17 \mathrm{~mJ} / \mathrm{cm}^{2}$; and (3) $\mathscr{T}$ and $\mathscr{R}$ are complementary to each other except at this second dip. Because the second dip appears in the cooling phase where the absorption ( $\mathscr{A}$ ) in $a$-Si can only decrease, ${ }^{23}$ we must conclude that neither $\mathscr{R}$ nor $\mathscr{A}$ can account for this drop in $\mathscr{T}$. We thus interpret this second drop in $\mathscr{T}$ as due to diffuse scattering of the probe light at the liquid-solid interface. This occurs when the liquid undergoes spontaneous nucleation at certain superheated temperature when the laser fluence gets above a certain threshold. This is analogous to the critical opalescence ${ }^{25}$ observed for light scattering in liquids heated closed to the critical point. Moreover, it is observed that the depth of the second dip in $\mathscr{T}$ increases with increasing laser fluences and it saturates only at relatively high-fluence values at which the $a$-Si surface may have been heated to a temperature much higher than that of the critical point of the liquid. We thus speculate two possibilities: (1) The pressure $(P)$ or the temperature $(T)$ or both these two parameters of the liquid may become supercritical ${ }^{26}$ at the interface; or (2) both $P$ and $T$ remain subcritical below a certain superheat limit, ${ }^{3,11,25}$ and the nucleation creates a very thin vapor layer which insulates the liquid from the $a$-Si surface. In addition, the time lag $(\tau \leqslant 1 \mu \mathrm{s})$ for this second dip to occur is comparable to that observed in the droplet explo-
TABLE I. Time lag for spontaneous nucleation to take place after the UV pulse for different liquids.

\begin{tabular}{lc}
\hline Liquids & $\begin{array}{c}\tau \\
\text { (ns) }\end{array}$ \\
\hline Isopropanol & 300 \\
Ethanol & 250 \\
Methanol & 250 \\
Water/isopropanol mixture $(70 \% / 30 \%)$ & 200 \\
Water & 150 \\
\hline \hline
\end{tabular}

sion experiments. ${ }^{11}$ For any difference, we attribute it to the fact that the present experiment involves heterogeneous nucleation as contrasted to previous homogeneous nucleation in droplet experiments. ${ }^{11}$ We further observed that $\tau$ is insensitive to the laser fluences we tried. Table I shows the values of $\tau$ for different liquids on the $0.2-\mu \mathrm{m} a-\mathrm{Si} \mathrm{film}$, from which we see that $\tau$ for isopropanol is the longest among the liquids studied. Comparison of the values for different liquids lead us to conclude that for liquids of high surface tension such as water, the wetting of the $a$-Si surface is quite poor and the nucleation is highly heterogeneous. ${ }^{27}$ This heterogeneous nucleation is known to be more efficient than homogeneous nucleation, which then accounts for the relatively short delay times $(\tau)$ for liquids like water. In fact, it can be seen from Table I that $\tau$ in general increases with the decrease of the surface tension of the liquids. Except for the case of pure isopropanol, explosion of the liquids is visibly observable for laser fluences somewhat above the spontaneous nucleation threshold. These are consistent with the established data in the literature $^{28}$ concerning the pressure of the superheated liquids, from which one finds that except for isopropanol, all the other liquids we studied can be superheated to a temperature with a pressure well above $1 \mathrm{~atm}$.

Figures 3(a)-3(c) show the corresponding data for the $1.0-\mu \mathrm{m}$ sample. For the two liquids we have studied in this case, ethanol and isopropanol/water mixture, the nucleation signatures are in general less pronounced than those that appeared for the thinner $(0.2-\mu \mathrm{m})$ sample. This is due to that the thermal diffusion length is much shorter than the sample thickness in this case and the transmission signal actually measures an integrated thermal effect through the sample. For the same reason, the relatively drop in $\mathscr{T}$ in this case is somewhat smaller for the same laser fluence. These points will be made more clear in the following when we come to the calibration of the results.

Figures 4 (a) and 4(b) show a collection of all the data for the two samples of different thicknesses. We have plotted for various pulsed UV laser fluences the probe transmittance dip $\left(\mathscr{T}_{n}\right)$ at the end of the UV pulse normalized to that at room temperature, i.e., before the UV pulse. Arrows A, B, and C in the figures indicate the thresholds for the occurrence of nucleation (second dip) and visibly observed explosive vaporization for some of the liquids as explained in the caption. For pure water, the two thresholds are practically indistinguishable. It is interesting to note that except for a few low-fluence data for the alcohols, 

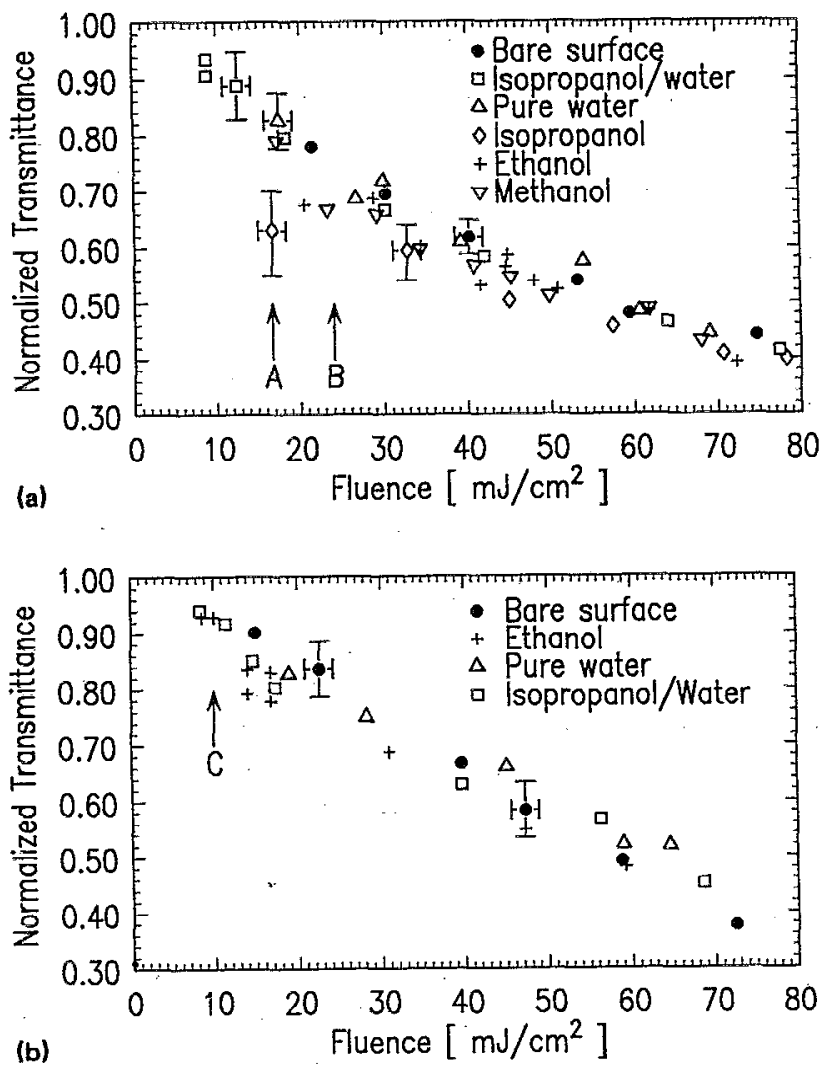

FIG. 4. Normalized transmittance plotted against laser fluence for bare and wet $a$-Si surfaces of (a) $0.2-\mu \mathrm{m}$ film and (b) $1.0-\mu \mathrm{m}$ film with different liquids used. Arrows $A$ and $B$ in (a) indicate the spontaneous nucleation threshold for isopropanol and the visibly detected explosion threshold for pure water film, respectively. Arrow $C$ in (b) indicates the spontaneous nucleation threshold for ethanol film. In the pure water case, the threshold for nucleation occurs very close to the explosion threshold.

the results for $\mathscr{T}_{n}$ exhibit a universal behavior for the different liquids. Furthermore, we have performed experiments to study the effects of the liquid-film thickness on our results. This is achieved by varying the delay time between the trigger for the puffer and the UV laser (see Fig. 1). This in turn controls the film thickness, as can be seen from the inset of Fig. 2. Figure 5 shows some results for $\mathscr{T}_{n}$ versus different delay times at a fixed fluence for isopropanol films on the 1- $\mu \mathrm{m}$ sample. As can be seen, with the delay time varied in a significant range, the measured $\mathscr{T}_{n}$ stays more or less constant within the error bars. Hence we can conclude that, within the present experimental accuracy, the liquid-film thickness has an insignificant effect on our measurement of $\mathscr{T}_{n}$. We believe that this reflects the fact that the reduced UV reflectance effect and thermal coupling effect due to the presence of the liquid film are roughly balanced out with each other, with the film quality not good enough to show a coherent interference effect of the UV light. This can also be checked from a crude estimate using calorimetric arguments. Taking all these into account and also the fact that the UV laser is transparent to all the liquids we studied, we can conclude that to first order of accuracy, we have to consider only the

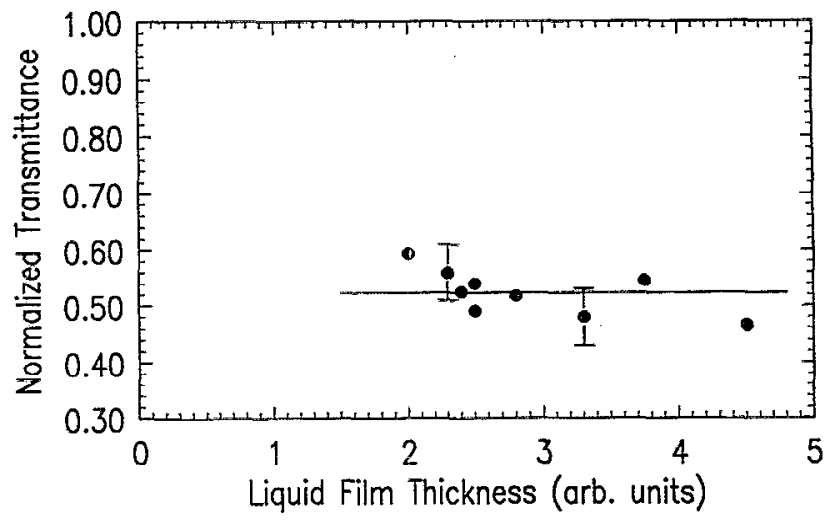

FIG. 5. Normalized transmittance dip plotted against the different thickness (varied in a random fashion) of isopropanol film on the $a$-Si surface at a fixed laser fluence.

$a$-Si film in the following photothermal analysis of our data for $\mathscr{T}_{n}$.

\section{DATA ANALYSIS}

In order to calibrate our results for $\mathscr{T}_{n}$ to obtain the interface temperatures, we have to take into account the effect of the temperature profile in the $a$-Si film due to the finite thermal diffusion length of the sample. Previously, ${ }^{23}$ in a steady-state experiment in which we have uniform heating for the $a$-Si film, we have obtained the temperature dependence of the optical constants ( $n$ and $k$ ) for the samples. To obtain the present calibration curves, we have to use these $n(T)$ and $k(T)$ to calculate $\mathscr{T}_{n}$ with the temperature profiles taken into account. To obtain these profiles, we resort to solutions from the one-dimensional (1D) heat diffusion equation:

$$
\rho C_{p} \frac{\partial T}{\partial t}=\frac{\partial}{\partial z}\left(K \frac{\partial T}{\partial z}\right)+Q(z, t)
$$

Here $\rho, C_{p}$, and $K$ are, respectively, the density, specific heat, and thermal conductivity of the $a-\mathrm{Si}$ film; and $Q(z, t)$ $=(1-R) I_{0}(t) \alpha e^{-\alpha z}$ is the source function with $R, \alpha$ being the reflectivity and absorption coefficient for $a-\mathrm{Si}$, respectively.

In order to solve (1), we have to know $I_{0}(t)$, which is the temporal profile of the UV pulse. Figure 6 shows a

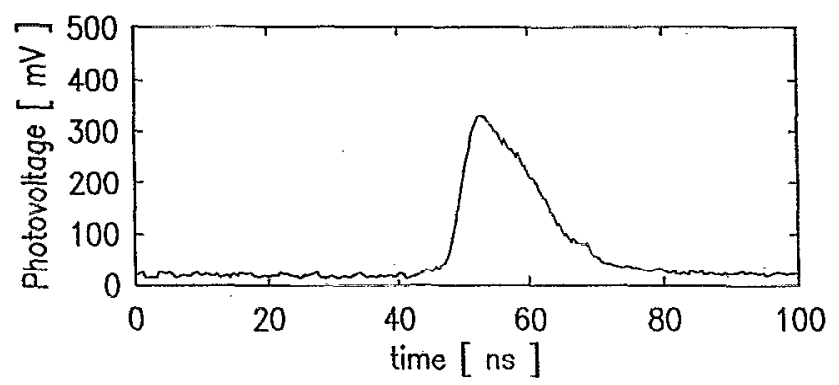

FIG. 6. Temporal profile of the $\mathrm{KrF}$ laser pulse which is modeled as triangular in shape in our data analysis of the experimental results. 
standard shape of our $\mathrm{KrF}$ laser pulse which we can fit using a triangular pulse shape:

$$
I_{0}(t)=\left\{\begin{array}{ccc}
\frac{2 F}{t_{1} t_{2}} t, & \text { for } & 0<t<t_{1}, \\
\frac{2 F}{t_{2}\left(t_{2}-t_{1}\right)}\left(t_{1}-t\right), & \text { for } & t_{1}<t<t_{2},
\end{array}\right.
$$

with $t_{1}=6 \mathrm{~ns}$ and $t_{2}=26 \mathrm{~ns}$, respectively. $F$ is the fluence of the pulse and we have shifted the time origin to about 48 ns. The optical absorption coefficient for $a-\mathrm{Si}$ at the $\mathrm{KrF}$ wavelength $(248 \mathrm{~nm})$ is taken as $1.43 \times 10^{-6} / \mathrm{cm}^{29}$ and the thermal constants are taken from the literature ${ }^{30}$ from which we found very minor temperature dependence for these constants. We are thus justified to take the average values for these constants within the temperature range considered for solving Eq. (1). ${ }^{31}$ Substituting Eq. (2) in Eq. (1), we first calculate the temporal profile for $T$ at the surface $(z=0)$ and find that $T$ peaks at about 15 ns after the incidence of the pulse. Then, assuming the deepest drop in $\mathscr{T}_{n}$ corresponds to the peak value of $T(z=0)$, we then calculate $T(z, t=15 \mathrm{~ns})$ as our temperature profile for calibration purpose. After obtaining this profile, we then approximate it by many thin slabs of different temperatures $T_{i}$ with the optical properties of each slab characterized by $n\left(T_{i}\right)$ and $k\left(T_{i}\right)$ as illustrated in Fig. 7.

To obtain the transmittance of the probe light through

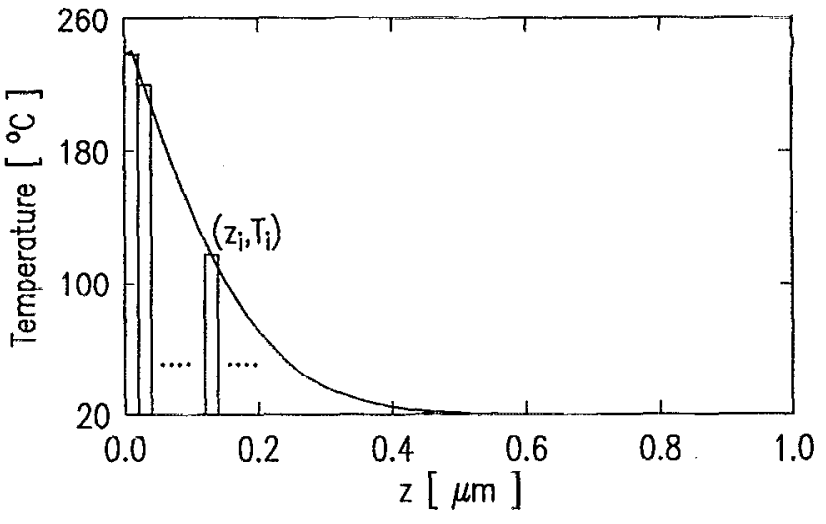

FIG. 7. Temperature profile of the $1.0-\mu \mathrm{m} a-\mathrm{Si}$ film as obtained from solving the $1 \mathrm{D}$ heat diffusion equation.

this collection of slabs, we start by writing down the characteristic matrix for each slab as ${ }^{32}$

$$
M_{i}=\left[\begin{array}{cc}
\cos \beta_{i} & -j \tilde{n}_{i} \cos \theta_{i} \sin \beta_{i} \\
-\frac{j}{\tilde{n}_{i}} \cos \theta_{i} \sin \beta_{i} & \cos \beta_{i}
\end{array}\right],
$$

where $j=\sqrt{-1}, \widetilde{n}_{i}=n_{i}+i k_{i}$, and $\theta_{i}$ is the angle of refraction in the $i$ th slab; $\beta_{i}=\left(2 \pi \widetilde{n}_{i} \Delta z_{i} / \lambda\right) \cos \theta_{i}$, and $\Delta z_{i}$ the slab thickness, $\lambda$ the probe wavelength, and ${ }^{23}$

$$
\begin{aligned}
& n_{i}=n\left(T_{i}\right)=\left\{\begin{array}{cc}
4.00+1.30 \times 10^{-4} \times\left(T_{i}-293\right), & \text { for the } 0.2-\mu \mathrm{m} \text { sample, } \\
4.07+5.00 \times 10^{-4} \times\left(T_{i}-293\right), & \text { for the } 1.0-\mu \mathrm{m} \text { sample, }
\end{array}\right. \\
& k_{i}=k\left(T_{i}\right)=\left\{\begin{array}{cc}
4.60 \times 10^{-2}+2.3 \times 10^{-4} \times\left(T_{i}-293\right), & \text { for the } 0.2-\mu \mathrm{m} \text { sample, } \\
5.48 \times 10^{-2}+1.46 \times 10^{-4} & \\
\times\left(T_{i}-273\right)+3.30 \times 10^{-7} \times\left(T_{i}-273\right)^{2}, & \text { for the } 1.0-\mu \mathrm{m} \text { sample. }
\end{array}\right.
\end{aligned}
$$

The overall characteristic matrix is then given by

$$
M=\prod_{i} M_{i}=\left[\begin{array}{ll}
M_{11} & M_{12} \\
M_{21} & M_{22}
\end{array}\right]
$$

and the transmittance by

$$
\mathscr{F}=\left|\frac{2 q}{\left(M_{11}+M_{12} q\right) q+\left(M_{21}+M_{22} q\right)}\right|^{2},
$$

with $q=\cos \theta_{0}, \theta_{0}$ being the angle of incidence. It is interesting to note that a similar profile analysis as the present one has been applied recently in studies of hydrogenated $a$-Si film where the profile for the absorption arises from the distribution of defects in the sample. ${ }^{33}$ Figures $8(\mathrm{a})$ and $8(\mathrm{~b})$ show our calibration curves obtained as described above for the two $a$-Si samples. We also show the result (the dotted line) in the uniform heating case for the $0.2-\mu \mathrm{m}$ sample obtained from the above fitted $n_{i}$ and $k_{i}$. This result fits very well the norminal values of the data obtained in our previous steady-state experiment except that the interference pattern is washed away due to the fitting process.

Using the solid curves in Figs. $8(\mathrm{a})$ and $8(\mathrm{~b})$, the results in Figs. 4(a) and 4(b) are calibrated for surface temperatures below $400{ }^{\circ} \mathrm{C}$. This is so restricted since our steady-state experiment ${ }^{23}$ was done only up to $400^{\circ} \mathrm{C}$, for other complications such as oxidation and polycrystallization will start at higher temperatures. ${ }^{34}$ Nevertheless, this range of temperature is enough for us to study the superheating of the liquids. Figures 9 (a) and 9(b) show the calibrated results for the two samples with the solid lines showing the theoretical calculations for the peak surface temperature of an infinite thick $a$-Si sample. We observe that within experimental error, most of the calibrated temperatures are in reasonable agreement with the calculated results. Two data points for isopropanol seem to show somewhat different behavior at low fluences, as can readily be seen from Figs. 4(a) and 4(b). We suspect that this may be due to the small signal-to-noise ratio at low flu- 

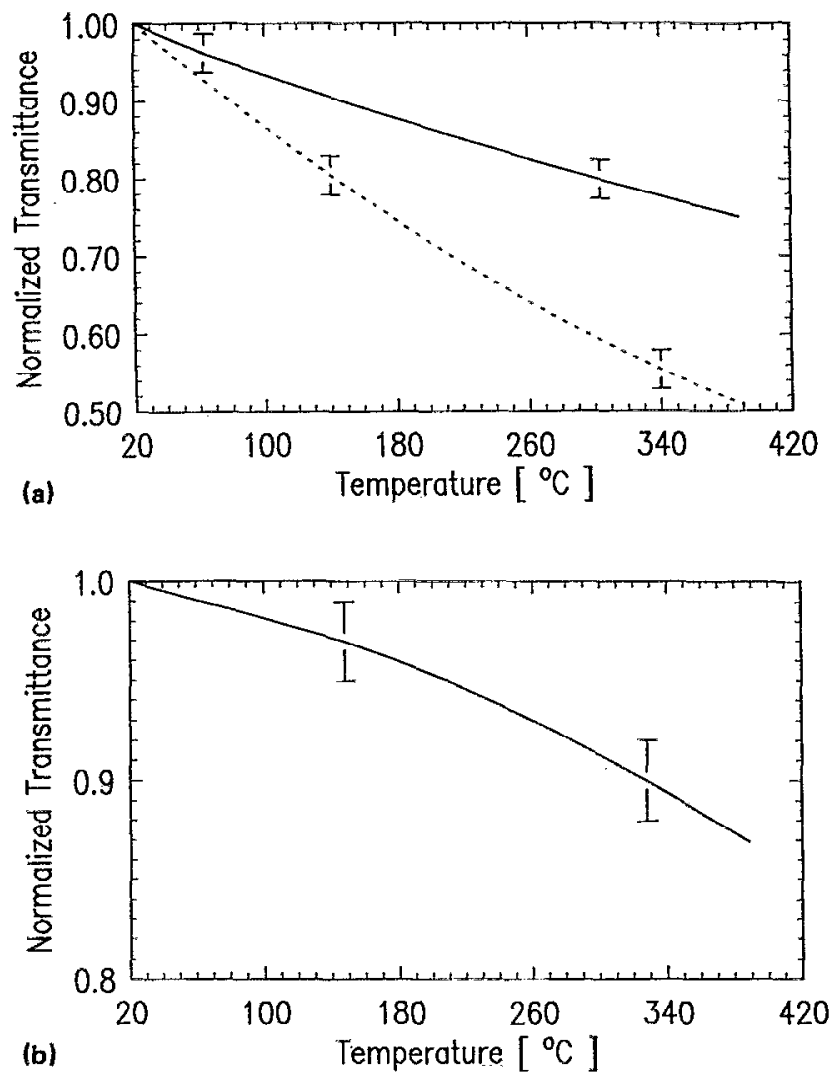

FIG. 8. Normalized transmittance vs temperature curves obtained by applying the rcsults fitted to our previous data from stcady heating experiments (Ref. 23). The solid curves in (a) and (b) are the results converted for transient calibration for the $0.2-$ and $1.0-\mu \mathrm{m}$ samples, respectively. The dotted curve in (a) shows the steady-state transmittance calculated from the $n(T)$ and $k(T)$ given in Eq. (4).

ences together with possible additional noise due to the fast evaporation rate for the heated alcohol. We also note that the agreement in the case for the $1.0-\mu \mathrm{m}$ sample is not as good, and we believe this is due to the larger temperature profiling effect in this case. The many approximations [such as in solving Eq. (1) and in fitting $n_{i}$ and $k_{i}$ ] introduced in the above profiling analysis simply leads to greater inaccuracy in the thick sample case. In addition, the threshold nucleation fluences from Figs. 4(a) and 4(b) are also indicated and they have values of 17,24 , and 10 $\mathrm{mJ} / \mathrm{cm}^{2}$, respectively, for isopropanol, water, and ethanol films. If we refer to the solid curves, we obtain the corresponding temperatures to be 210,290 , and $140^{\circ} \mathrm{C}$, respectively. These are in reasonable agreement with the data from literature which have values approximately to be 220 , 307 , and $195^{\circ} \mathrm{C}$ for the three pure liquids. ${ }^{28} \mathrm{We}$ should also remark that our identification of the deepest drop in $\mathscr{T}$ as the signature for the hottest surface temperature may introduce certain inaccuracy, since $\mathscr{T}$ measures the average temperature over the sample, and the hottest surface temperature may occur at a time slightly earlier than that for this largest drop. A rough estimate shows that this can lower the calibrated temperature values by as much as $10 \%$. Hence, we conclude that within experimental error, the present measurements and calibrated results, the theo-
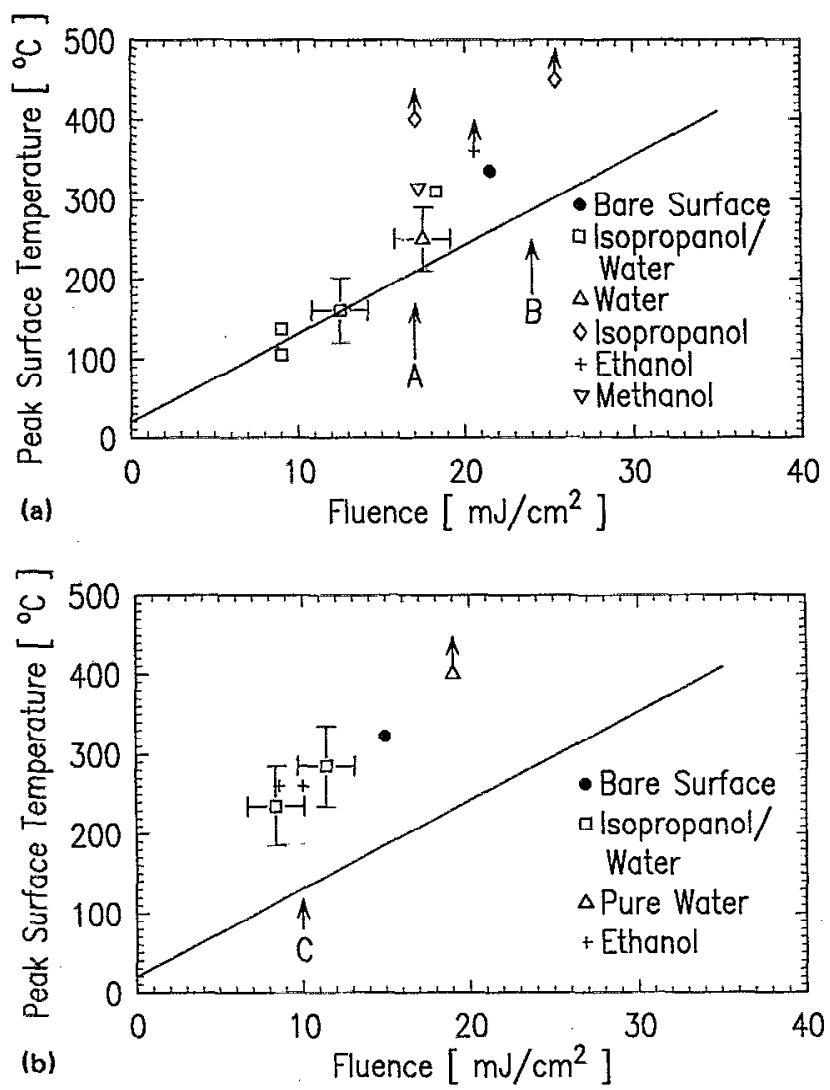

FIG. 9. Comparison between calibrated results (from Figs. 4 and 8 ) with thcoretical calculations. The arrows indicate the thresholds as in Fig. 4. In (a) the peak temperature data derived for isopropanol and ethanol for the $0.2-\mu \mathrm{m} a-\mathrm{Si}$ sample go beyond the scale, and so does the peak temperature derived for pure water in (b) for the 1.0- $\mu \mathrm{m}$ a-Si sample.

retical calculations, and the concept of strongly superheated liquid film at the interface are all consistent with each other.

\section{CONCLUSION}

Through the application of an optical transmission technique, we have been able to monitor the temperature and nucleation dynamics of an explosively superheated liquid film in the vicinity of an opaque substrate. Results obtained support the picture for a strongly superheated thin liquid layer at the interface upon the absorption of the excimer laser pulse ( $\sim 10 \mathrm{~ns})$ by the substrate. Huge transient liquid pressures are then generated at the interface, which provide for a mechanism for particulate removal in an actual laser cleaning process. ${ }^{5-8}$ We conclude that factors like the surface tension of the liquid are crucial where others like the liquid-film thickness are relatively insignificant in such film explosion arrangements. In addition, our approach provides further information concerning the nucleation dynamics of the interfacial liquid through the appearance of a second dip in the transmission signal. This second transmission dip may be related to critical opalescence, heterogeneous nucleation, and possible supercritical heating, which we feel is of great interest and worthy of further investigations. If the superheated liquid is really 
insulated from the substrate surface as we speculated, then we have to use a method to probe its temperature other than transmission probing of the substrate temperature, as done here.

\section{ACKNOWLEDGMENTS}

We thank Professor Costas Grigoropoulos of the University of California at Berkeley for useful discussions. PTL acknowledges support from the Faculty Development Funding of the Portland State University.

'See, e.g., V. P. Skripov, E. N. Sinitsyn, P. A. Pavlov, G. V. Ermakov, G. N. Muratov, N. V. Bulanov, and V. G. Baidakov, Thermalphysical Properties of Liquids in the Metastable State (Gordon and Breach, New York, 1988).

${ }^{2}$ F. V. Bunkin, JETP Lett. 19, 175 (1974). The term "optothermodynamics" describing this field of study was first coined in this paper.

${ }^{3}$ For the latest work, see, e.g., H. Latifi, J. G. Xie, T. E. Ruekgauer, R. L. Armstrong, and R. B. Pinnick, Opt. Lett. 16, 1129 (1991).

${ }^{4}$ J. D. Kelley, M. I. Stuff, F. E. Hovis, and G. J. Linford, SPIE Proc. 1415, 211 (1991).

${ }^{5}$ W. Zapka, K. Asch, J. Keyser, and K. Meissner, "Removal of Particles from Solid-State Surfaces," European patent EP 0297506 A2, Jan. 4, 1989.

${ }^{6}$ W. Zapka, and A. C. Tam, Conference on Lasers and Electro-Optics, CLEO '90 Conference Digest (Optical Society of America, Washington, DC, 1990), p. 226.

${ }^{7}$ W. Zapka, W. Ziemlich, and A. C. Tam, Appl. Phys. Lett. 58, 2217 (1991).

${ }^{8}$ A. C. Tam, W. P. Leung, W. Zapka, and W. Ziemlich, J. Appl. Phys. 71, 3515 (1992).

${ }^{9}$ K. Imen, S. J. Lee, and S. D. Allen, Conference on Lasers and ElectroOptics, CLEO '90 Conference Digest (Optical Society of America, Washington, DC, 1990), p. 228.

${ }^{10}$ K. Imen, S. J. Lee, and S. D. Allen, Appl. Phys. Lett. 58, 203 (1991).

"B. S. Park, A. Biswas, R. L. Armstrong, and R. G. Pinnick, Opt. Lett. 15, 206 (1990), and references therein.

${ }^{12}$ See, e.g., H. G. Dreehsen, C. Hartwich, J. H. Schaefer, and J. Uhlenbusch, J. Appl. Phys. 56, 238 (1984).

${ }^{13}$ See, e.g., G. E. Jellison, Jr., in Semiconductors and Semimetals (Academic, New York, 1984), Vol. 23, and references therein. See also G. E. Jellison, Jr. and H. H. Burke, J. Appl. Phys. 60, 841 (1986) and M. Bertolotti, V. Bogdanov, A. Ferrari, A. Jascow, N. Nazorova, A. Pikhtin, and L. Schirone, J. Opt. Soc. Am. B 7, 918 (1990).
${ }^{14}$ See, e.g., M. E. Thomas, in Handbook of Optical Constants of Solids II, edited by E. D. Palik (Academic, New York, 1991), p. 177, and references therein.

${ }^{15}$ See the papers by G. E. Jellison, Jr. and co-workers in Ref. 9, and references therein.

${ }^{16}$ See, e.g., A. Aydinli, H. W. Lo, M. C. Lee, and A. Compaan, Phys. Rev. Lett. 46, 1640 (1981); J. M. Liu, H. Kurz, and N. Bloembergen, Appl. Phys. Lett. 41, 643 (1982).

${ }^{17}$ J. C. Strum, P. V. Schwartz, and P. M. Garone, Appl. Phys. Lett. 56, 961 (1990).

${ }^{18}$ P. J. Timmans, R. A. McMahon, and H. Ahmed, Appl. Phys. Lett. 53, 1844 (1988)

${ }^{19}$ L. A. Lompre, J. M. Liu, H. Kurz, and N. Bloembergen, Appl. Phys. Lett. 43, 168 (1983).

${ }^{20}$ L. Klees, P. T. Leung, N. Do, F. Tong, W. P. Leung, and A. C. Tam, Conference on Lasers and Electro-Optics CLEO '92 Conference Digest (Optical Society of America, Washington, DC, 1992), p. 502.

${ }^{21}$ See, e.g., E. P. Donovan, F. Spaepen, D. Turnbull, J. M. Poate, and D. C. Jacobson, Appl. Phys. Lett. 42, 698 (1983); J. Narayan, C. W. White, M. J. Aziz, B. Stritzker, and A. Walthuis, J. Appl. Phys. 57, 564 (1985).

${ }^{22}$ M. R. T. Siregar, M. von Allmen, and W. Luthy, Helv. Phys. Acta 52, 45 (1979); M. von Allmen, W. Luthy, J, P. Thomas, M. Fallavier, J. M. Mackowski, R. Kirsch, M. A. Nicolet, and M. E. Roulet, Appl. Phys. Lett. 34, 82 (1979); and C. K. Ong, H. S. Tan, and E. H. Sin, Mater. Sci. Eng. 79, 79 (1986).

${ }^{23}$ N. Do, L. Klees, P. T. Leung, F. Tong, W. P. Leung, and A. C. Tam, Appl. Phys. Lett. 60, 2186 (1992).

${ }^{24}$ The nitridation of Si occurs at a much higher temperature. See, e.g., $\mathrm{H}$. Nakamura, M. Kaneko, and S. Matsumoto, Appl. Phys. Lett. 43, 691 (1983)

${ }^{25}$ V. P. Skripov, Metastable Liquids (Wiley, New York, 1974).

${ }^{26}$ F. V. Bunkin and M. I. Tribel'skii, Sov. Phys. Usp. 23, 105 (1980).

${ }^{27}$ M. Blander and J. L. Katz, AIChE J. 21, 833 (1975).

${ }^{28}$ C. T. Avedisian, J. Phys. Chem. Ref. Data 14, 695 (1985).

${ }^{29}$ E. D. Palik, Ed., Handbook of Optical Constants of Solids I (Academic, New York, 1985), p. 571.

${ }^{30}$ See the paper by C. K. Ong, H. S. Tan, and E. H. Sin in Ref. 22, and references therein.

${ }^{31}$ The authors want to thank Professors C. Grigoropoulos, S. M. George, and Dr. B. Koehler for correspondence on this.

${ }^{32} \mathrm{M}$. Born and E. Wolf, Principle of Optics, 6th ed. (Pergamon, New York, 1980); R. Jacobsson, Progress in Optics, edited by E. Wolf (North-Holland, Amsterdam, 1965), Vol. 5, p. 247.

${ }^{33}$ A. Asano and M. Stutzmann, J. Appl. Phys. 70, 5025 (1991).

${ }^{34}$ Damage of the $a$-Si sample can also occur at higher temperatures; this could be detected at fluences above $80 \mathrm{~mJ} / \mathrm{cm}^{2}$ in our experiment. 